\title{
ON A LOCAL DEGREE FOR A CLASS OF MULTI-VALUED VECTOR FIELDS IN INFINITE DIMENSIONAL BANACH SPACES
}

\author{
N. M. BENKAFADAR AND B. D. GEL'MAN
}

\begin{abstract}
This paper is devoted to the development of a local degree for multi-valued vector fields of the form $f-F$. Here, $f$ is a single-valued, proper, nonlinear, Fredholm, $C^{1}$-mapping of index zero and $F$ is a multivalued upper semicontinuous, admissible, compact mapping with compact images. The mappings $f$ and $F$ are acting from a subset of a Banach space $E$ into another Banach space $E_{1}$. This local degree is used to investigate the existence of solutions of a certain class of operator inclusions.
\end{abstract}

\section{INTRODUCTION}

An important part of the theory of multi-valued mappings is devoted to inclusions of the type $f(x) \in F(x)$, where $f$ is a single-valued mapping and $F$ is a multi-valued mapping. Such inclusion types can be found in different branches of mathematics, e.g., optimal control problems, mathematical economics, game theory.

For the solution of such problems we often employ topological invariant methods. In particular, we use the theory of topological degree, the rotation of a vector field, etc.

In this paper we study the following case: $f$ is a nonlinear Fredholm mapping and $F$ is an admissible, compact, multi-valued mapping. For this purpose we introduce the concept of the local topological degree for mappings of the form $f-F$. We thus generalize the results given by the authors in [2].

For the construction of the local degree we study the degree of an admissible $*$-pair $(p ; q)$. This degree is defined for mappings which are acting

1991 Mathematics Subject Classification. 47H04, 47H11, 47H15.

Key words and phrases. Local degree, nonlinear Fredholm mapping, multi-valued mapping, operator inclusion, homology group.

The second author acknowledges support by the Russian Fund of Fundamental Research \#96-01-00360.

Received: June 17, 1996. 
from a finite-dimensional manifold to a finite-dimensional vector space. This process generalizes the construction of the coincidence index which has been introduced by Kucharski [13].

A topological degree for mappings $f-F$ in the case when $F$ is a compact multi-valued mappings with convex images has been constructed in the article of Borisovich [3]. When the multi-valued mapping $F$ is condensing relatively to the mapping $f$, a topological degree has been constructed in the papers [4] and [5].

The local degree constructed in this paper is employed to investigate the existence of solutions of a class of operator inclusions.

\section{A DEGREE FOR ADMISSIBLE $*$-PAIRS IN THE FINITE-DIMENSIONAL CASE}

Let $(X, A)$ and $(Y, B)$ be two pairs of Hausdorff topological spaces, and let $p:(Y, B) \rightarrow(X, A)$ be a continuous single-valued mapping.

Definition 1.1. The mapping $p$ is called a "Vietoris" mapping if

1. $p$ is proper and surjective;

2. $p^{-1}(x)$ is acyclic for every $x \in X$.

The mapping $p$ induces the homomorphisms $p_{*}$ of the Cech homology groups with compact support and coefficients in $Q$. For more information about the Čech homology groups, the reader is referred to [17].

Theorem 1.2. [11] If $p:(Y, B) \longrightarrow(X, A)$ is a Vietoris mapping, then the homomorphism $p_{*}: H(Y, B) \longrightarrow H(X, A)$ is an isomorphism.

Definition 1.3. A pair of mappings $(p ; q)$ which satisfies:

1. $p:(Y, B) \longrightarrow(X, A)$ is a Vietoris mapping,

2. $q:(Y, B) \longrightarrow(Z, C)$ is a continuous mapping

is called $*$-pair.

Let $M^{n}$ be a $n$-dimensional manifold, $R^{n}$ a $n$-dimensional topological vector space and $X$ a topological space. Let $p$ and $q$ be two single-valued mappings of the form :

$$
M^{n} \stackrel{p}{\longleftarrow} X \stackrel{q}{\longrightarrow} R^{n} .
$$

The pair $(p ; q)$ is called an "admissible $*$-pair" if the subset $K=p \circ q^{-1}(\theta)$ of $M^{n}$ is compact.

We shall give a construction of a local degree for this class of pairs of single-valued mappings.

Consider an admissible $*$-pair $(p ; q)$. Then we have

$$
\left(M^{n} ; M^{n} \backslash K\right) \stackrel{p}{\longleftarrow}\left(X ; X \backslash q^{-1}(\theta)\right) \stackrel{q}{\longrightarrow}\left(R^{n} ; R^{n} \backslash \theta\right),
$$

and we can thus deduce the following diagram:

$$
H_{n}\left(M^{n} ; M^{n} \backslash K\right) \stackrel{p_{*}}{\longleftarrow} H_{n}\left(X ; X \backslash q^{-1}(\theta)\right) \stackrel{q_{*}}{\longrightarrow} H_{n}\left(R^{n} ; R^{n} \backslash \theta\right) .
$$

Using Theorem 1.2, we confirm that $p_{*}$ is an isomorphism and we can thus define the homomorphism

$$
(p ; q)_{*}=q_{*} \circ p_{*}^{-1}: H_{n}\left(M_{n} ; M_{n} \backslash K\right) \longrightarrow H_{n}\left(R^{n} ; R^{n} \backslash \theta\right) .
$$


Suppose now that $M^{n}$ is oriented with a fixed orientation $O \in \Gamma\left(M^{n} ; Q\right)$. Let $O_{K}$ be the fundamental class of the compact set $K$ and let $O_{\theta}$ be the fundamental class of the zero $\theta \in R^{n}$. The homomorphism $(p ; q)_{*}$ transforms $O_{K}$ into $O_{\theta}$ multiplied by an integer $\gamma_{\theta}(p ; q)$. Thus,

$$
(p ; q)_{*}\left(O_{K}\right)=q_{*} \circ p_{*}^{-1}\left(O_{K}\right)=\gamma_{\theta}(p ; q) \cdot\left(O_{\theta}\right) .
$$

Definition 1.4. The integer $\gamma_{\theta}(p ; q)$, defined above, is called the "local degree" of the admissible *-pair $(p ; q)$.

In some particular cases we will denote this degree by $\gamma_{\theta}\left[(p ; q), M^{n}\right]$.

We now consider some properties of this degree. that

Let $(p ; q)$ be an admissible *-pair and $K_{1}$ a compact subset of $M^{n}$ such

$$
K=p \circ q^{-1}(\theta) \subset K_{1} .
$$

We consider the mappings

$$
\left(M^{n} ; M^{n} \backslash K_{1}\right) \stackrel{\tilde{p}}{\longleftarrow}\left(X ; X \backslash p^{-1}\left(K_{1}\right)\right) \stackrel{\tilde{q}}{\longrightarrow}\left(R^{n} ; R^{n} \backslash \theta\right),
$$

where $\tilde{p}$ and $\tilde{q}$ are the restrictions of $p$ and $q$, respectively.

Proposition 1.5. The homomorphism $(\tilde{p} ; \tilde{q})_{*}$ transforms the fundamental class $O_{K_{1}}$ into the fundamental class $O_{\theta}$ multiplied by $\gamma_{\theta}(p ; q)$.

The proof of this fact is a consequence of the commutativity of the corresponding diagram.

Definition 1.6 (Homotopy). Two admissible $*$-pairs $\left(p_{0} ; q_{0}\right)$ and $\left(p_{1} ; q_{1}\right)$ such that

$$
M^{n} \stackrel{p_{i}}{\longleftarrow} X_{i} \stackrel{q_{i}}{\longrightarrow} R^{n}, \quad i=0,1,
$$

are called "homotopic" if and only if the following conditions are satisfied:

1. there exists an admissible $*$-pair $(p ; q)$ such that

$$
M^{n} \times[0,1] \stackrel{p}{\longleftarrow} X \stackrel{q}{\longrightarrow} R^{n} ;
$$

2. there exist two continuous mappings such that

$$
h_{i}: X_{i} \longrightarrow X ; \quad i=0,1,
$$

and the following diagram is commutative:

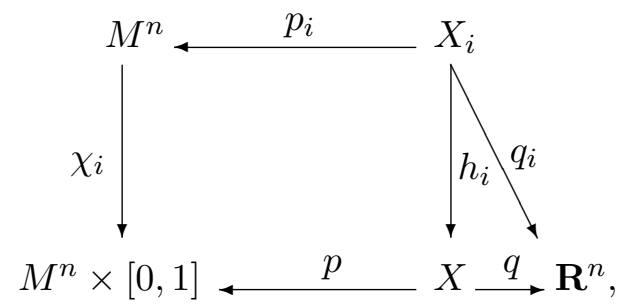

where $\chi_{i}(x)=(x, i), i=0,1$. 
Proposition 1.7 (Homotopy Invariance). Let $\left(p_{\alpha} ; q_{\alpha}\right), \alpha=0,1$, be two admissible *-pairs which are homotopic. Let $(p ; q)$ be the homotopy connecting them such that

$$
M_{n} \times[0,1] \stackrel{p}{\longleftarrow} X \stackrel{q}{\longrightarrow} R^{n} .
$$

If $K=\cup_{\lambda \in[0,1]} K_{\lambda}$ is compact and $K_{\lambda}=p_{\lambda} \circ q_{\lambda}^{-1}(\theta)$, where $p_{\lambda}$ and $q_{\lambda}$ are the restrictions of $p$ and $q$ on the set $p^{-1}\left(M^{n} \times \lambda\right)$ respectively, then $\gamma_{\theta}\left(p_{0} ; q_{0}\right)=$ $\gamma_{\theta}\left(p_{1} ; q_{1}\right)$.

The proof of this proposition is an analogue of the same theorem in [13].

Proposition 1.8 (Product). Let $\left(p_{1} ; q_{1}\right)$ and $\left(p_{2} ; q_{2}\right)$ be two admissible *pairs of the forms

$$
M_{1}^{n} \stackrel{p_{1}}{\longleftarrow} X_{1} \stackrel{q_{1}}{\longrightarrow} R^{n} \quad \text { and } \quad M_{2}^{m} \stackrel{p_{2}}{\longleftarrow} X_{2} \stackrel{q_{2}}{\longrightarrow} R^{m},
$$

respectively. If

$$
M_{1}^{n} \times M_{2}^{m} \stackrel{p_{1} \times p_{2}}{\longleftarrow} X_{1} \times X_{2} \stackrel{q_{1} \times q_{2}}{\longrightarrow} R^{n} \times R^{m},
$$

then $\left(p_{1} \times p_{2} ; q_{1} \times q_{2}\right)$ is also an admissible $*$-pair and

$$
\gamma_{\theta}\left(p_{1} \times p_{2} ; q_{1} \times q_{2}\right)=\gamma_{\theta}\left(p_{1} ; q_{1}\right) \cdot \gamma_{\theta}\left(p_{2} ; q_{2}\right) .
$$

The proof of this proposition is a consequence of the formula of Kunetta and the corresponding commutative diagram.

Let $M^{n}$ be an oriented manifold of class $C^{1}, L^{n-1}$ an oriented submanifold $M^{n}$ of class $C^{1}$ and $(p ; q)$ an admissible $*$-pair with

$$
M^{n} \stackrel{p}{\longleftarrow} X \stackrel{q}{\longrightarrow} R^{n},
$$

where $K=p \circ q^{-1}(\theta) \subset L^{n-1}$ and $q\left(p^{-1}\left(L^{n-1}\right)\right) \subset R^{n-1} \subset R^{n}$.

Consider $(\tilde{p} ; \tilde{q})$, the restriction of $(p ; q)$ on $L^{n-1}$, i.e.,

$$
L^{n-1} \stackrel{\tilde{p}}{\longleftarrow} \tilde{X}=p^{-1}\left(L^{n-1}\right) \stackrel{\tilde{q}}{\longrightarrow} R^{n-1},
$$

where $\tilde{p}$ and $\tilde{q}$ are the restrictions of $p$ and $q$, respectively.

Proposition 1.9 (Restriction). The $*$-pair $(\tilde{p} ; \tilde{q})$ is an admissible *-pair. So, with the correct choice of the orientation, $\gamma_{\theta}(p ; q)=\gamma_{\theta}(\tilde{p} ; \tilde{q})$.

For this purpose we shall prove the following.

Lemma 1.10. Let $M^{n}$ be an oriented manifold of class $C^{1}$, and $L^{n-1}$ an oriented $C^{1}$-submanifold in $M^{n}$. Then for every compact $K \subset L^{n-1} \subset M^{n}$ there exists an open neighborhood $W \subset M^{n}$ such that $W$ is homeomorphic to the direct product $V \times(-\varepsilon, \varepsilon)$ and the homeomorphism between $V$ and $V \times\{0\}$ is the identity mapping, where $V=W \cap L^{n-1}$.

Proof. This is a consequence of the fact that there exists a neighborhood $U$ of the null selector of the normal bundle on $L^{n-1}$ and a homeomorphism $h$ of $U$ in some neighborhood of $L^{n-1}$ in $M^{n}$ such that $h(x, 0)=x$ for every $x \in L^{n-1}$ (see [14]). Because every line bundle on an oriented manifold is a product bundle [12], we can easily deduce the lemma. 
Proof of Proposition 1.9. Let $W$ be a neighborhood of the compact set $K=p_{\circ} q^{-1}(\theta)$ which satisfies the conditions of Lemma 1.10. The inclusion mapping $(W ; W \backslash K) \stackrel{i}{\longrightarrow}\left(M^{n} ; M^{n} \backslash K\right)$ induces an isomorphism of homology groups. Then $O_{K}\left(M^{n}\right)$ (the fundamental class in $M^{n}$ near $K$ ) is the image of $O_{K}(W)$ (the fundamental class in $W$ near $K$ ) with a suitable choice of orientation.

So, using the the excision property, we have $\gamma_{\theta}\left[(p ; q) ; M^{n}\right]=\gamma_{\theta}[(p ; q) ; W]$ and $\gamma_{\theta}\left[(\tilde{p} ; \tilde{q}) ; L^{n-1}\right]=\gamma_{\theta}[(\tilde{p} ; \tilde{q}) ; V]$.

Because there exists a homeomorphism $h: W \longrightarrow V \times(-\varepsilon, \varepsilon)$ we can consider the orientation $O(V \times(-\varepsilon, \varepsilon))$ in $V \times(-\varepsilon, \varepsilon)$ which is induced by the homeomorphism $h$.

Consider the admissible $*$-pair $\left(p_{1} ; q_{1}\right)$ :

$$
V \times(-\varepsilon, \varepsilon) \stackrel{p_{1}=h_{0}^{-1}}{\longleftarrow} X_{1}=p^{-1}(W) \stackrel{q_{1}=\left.q\right|_{X}}{\longrightarrow} R^{n} .
$$

Then $\gamma_{\theta}[(p ; q) ; W]=\gamma_{\theta}\left(p_{1}, q_{1}\right)$. Choose an orientation in $R^{n-1}$ and $R^{1}$ such that the product of the orientations coincides with the orientation in $R^{n}$. Let us consider the restriction of the orientation of $R^{1}$ on $(-\varepsilon, \varepsilon)$ and define an orientation on $\mathrm{V}$ such that the product of these orientations coincides with the orientation on $O(V \times(-\varepsilon, \varepsilon))$.

Let us calculate $\gamma_{\theta}\left(p_{1} ; q_{1}\right)$. Consider the following admissible $*$-pairs:

$$
V \times\{0\} \stackrel{h}{\approx} V \stackrel{\tilde{p}}{\stackrel{\tilde{X}}{\stackrel{\tilde{q}}{\longrightarrow}}} R^{n-1}
$$

and

$$
V \times(-\varepsilon, \varepsilon) \stackrel{\tilde{p} \times i d}{\longleftarrow} \widetilde{X} \times(-\varepsilon, \varepsilon) \stackrel{\tilde{q} \times i d}{\longrightarrow} R^{n-1} \times R^{1}=R^{n} .
$$

From the product property we have $\gamma_{\theta}(\tilde{p} \times i d ; \tilde{q} \times i d)=\gamma_{\theta}(\tilde{p}, \tilde{q}) \cdot \gamma_{\theta}(i d, i d)$. But, with our choice of the orientation, we have $\gamma_{\theta}(i d, i d)=1$. Then we deduce $\gamma_{\theta}(\tilde{p} \times i d ; \tilde{q} \times i d)=\gamma_{\theta}(\tilde{p} ; \tilde{q})$.

Consider the following diagram:

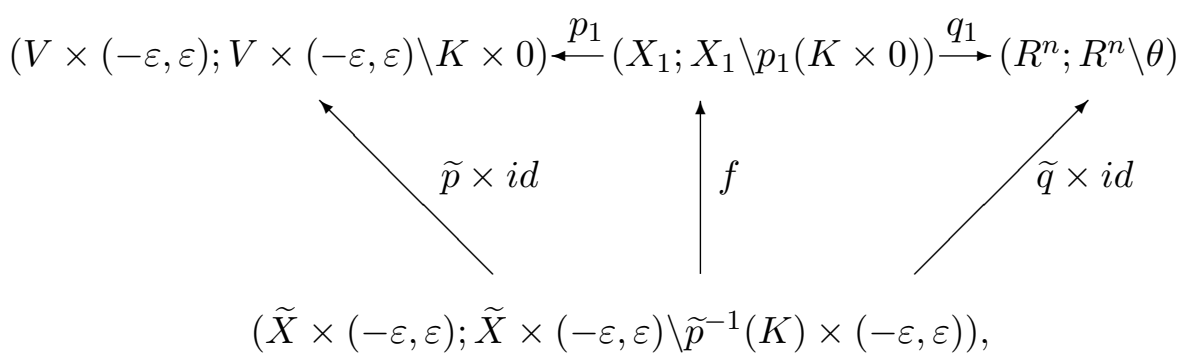

where $f(y, t)=y$ for every $(y, t) \in \tilde{X} \times(-\varepsilon, \varepsilon)$.

So we obtain a commutative diagram with the group's homologies induced by the above diagram. This is a consequence of the fact that $\tilde{p} \times i d$ is homotopic to $p_{1} \circ f$ and $\tilde{q} \times i d$ is homotopic to $q_{1} \circ f$. 
Using the commutativity of the corresponding diagram we obtain:

$$
\gamma_{\theta}(p ; q)=\gamma_{\theta}\left(p_{1} ; q_{1}\right)=\gamma_{\theta}(\tilde{p} \times i d ; \tilde{q} \times i d)=\gamma_{\theta}(\tilde{p} ; \tilde{q})
$$

Proposition 1.11. Let $(p ; q)$ be an admissible $*$-pair. If $\gamma_{\theta}(p ; q) \neq 0$, then there exist an element $x_{0} \in M^{n}$ such that $\theta \in q \circ p^{-1}\left(x_{0}\right)$.

This is a consequence of the construction of the local degree.

Proposition 1.12. Let $(p ; q)$ be an admissible *-pair, $M^{n} \stackrel{p}{\longleftarrow} X \stackrel{q}{\longrightarrow} R^{n}$, and suppose that there exists a connected neighborhood $W$ of $\theta$ in $R^{n}$ such that $q^{-1}(y)$ is compact. Then $\gamma_{\theta}(p ; q)=\gamma_{y}(p ; q)$ for every $y \in W$.

For the proof see [10].

We finish this section with the following proposition.

Proposition 1.13. Let $(p ; q)$ be an admissible $*$-pair such that

$$
M^{n} \stackrel{p}{\longleftarrow} X \stackrel{q}{\longrightarrow} R^{n}
$$

and let $U$ be an open subset of $M^{n}$ which contains the set $K=p \circ q^{-1}(\theta)$. Then

$$
\gamma_{\theta}\left[(p ; q) ; M^{n}\right]=\gamma_{\theta}[(p ; q) ; U] .
$$

It is easy to see that in the case $p=i d: M^{n} \longrightarrow M^{n}$ the local degree $\gamma_{\theta}(p ; q)$ coincides with the degree of Dold [10].

\section{Degree for a MUlti-VAlued Vector Field iN \\ A FINITE-DIMENSIONAL MANIFOLD}

Let $M^{n}$ be an oriented manifold with a fixed orientation $O \in \Gamma\left(M^{n} ; Q\right)$, and let $R^{n}$ be a $n$-dimensional topological vector space.

Let $\Phi$ be an upper semicontinuous multi-valued mapping with nonempty compact images such that $\Phi: M^{n} \longrightarrow K\left(R^{n}\right)$, where $K\left(R^{n}\right)$ is the set of all nonempty compact subsets of $R^{n}$. The properties of multi-valued mappings can be found in [8], [1].

Definition 2.1. The multi-valued mapping $\Phi$ is called an "admissible multivalued vector field" if there exists an admissible *-pair $(p ; q)$ such that

1. $M^{n} \stackrel{p}{\longleftarrow} X \stackrel{q}{\longrightarrow} R^{n}$;

2. $q \circ p^{-1}(x) \subset \Phi(x)$ for every $x \in M^{n}$;

3. The set $K=\left\{x \in M^{n} \mid \theta \in \Phi(x)\right\}$ of $M^{n}$ is compact.

In this case the pair of single-valued mappings $(p, q)$ is called a "selected pair" of $\Phi$ and we use the notation $(p, q) \subset \Phi$.

Definition 2.2. Let $\Phi: M^{n} \longrightarrow K\left(R^{n}\right)$ be an admissible multi-valued vector field. Then the set of integers

$$
\operatorname{Deg}_{\theta}\left(\Phi, M^{n}\right)=\left\{\gamma_{\theta}(p, q) \mid(p, q) \subset \Phi\right\}
$$

is called the "degree" of $\Phi$.

Let us now give some properties of this degree. 
Proposition 2.3. If $\Phi$ is an admissible multi-valued vector field with acyclic images then $\operatorname{Deg}_{\theta}\left(\Phi, M^{n}\right)$ is a singleton.

The proof of this proposition can be deduced from the theorem of Vietoris and the commutativity of the corresponding diagram.

Proposition 2.4. If $\operatorname{Deg}_{\theta}\left(\Phi, M^{n}\right) \neq\{0\}$, then there exists an element $x_{0} \in$ $M^{n}$ such that $\theta \in \Phi\left(x_{0}\right)$.

The proof of this proposition is a direct consequence of the definition of $\operatorname{Deg}_{\theta}\left(\Phi, M^{n}\right)$.

Proposition 2.5. Let $\Psi: M^{n} \times[0,1] \longrightarrow K\left(R^{n}\right)$ be a an admissible upper semicontinuous multi-valued mapping such that

$$
\nabla=\left\{x \in M^{n} \mid \theta \in \Psi(x, t) ; t \in[0,1]\right\}
$$

is a compact subset of $M^{n}$. Then $\Phi_{0}=\Psi(\cdot, 0)$ and $\Phi_{1}=\Psi(\cdot, 1)$ are admissible multi-valued vector fields and

$$
\operatorname{Deg}_{\theta}\left(\Phi_{0}, M^{n}\right) \cap \operatorname{Deg}_{\theta}\left(\Phi_{1}, M^{n}\right) \neq \emptyset .
$$

This is a direct consequence of the property of homotopy invariance 1.7.

Proposition 2.6. Let $M^{n}$ be an oriented manifold of class $C^{1}$ and $L^{n-1}$ an oriented submanifold in $M^{n}$. Let $\Phi: M^{n} \longrightarrow K\left(R^{n}\right)$ be an admissible multi-valued vector field such that

1. $K=\left\{x \in M^{n} \mid \theta \in \Phi(x)\right\} \subset L^{n-1}$;

2. $\tilde{\Phi}=\left.\Phi\right|_{L^{n-1}}: L^{n-1} \longrightarrow K\left(R^{n-1}\right)$.

Then $\operatorname{Deg}_{\theta}\left(\Phi, M^{n}\right) \subset \operatorname{Deg}_{\theta}\left(\tilde{\Phi}, L^{n-1}\right)$.

This proposition is a consequence of the property 1.9 .

3. A Degree for a multi-VAlued Vector field Perturbed By A FREDHOLM MAPPING

Let $E$ and $E_{1}$ be two Banach spaces, and let $U$ be an open bounded domain in $E$. Let $f: \bar{U} \longrightarrow E_{1}$ be a single-valued, proper, continuous mapping such that the restriction $\left.f\right|_{U}$ is a nonlinear Fredholm mapping with index zero of class $C^{1}$. We note that $f \in \Phi_{0} C^{1}$. The definition and properties of nonlinear Fredholm mappings can be found in $[9]$

Let $F: \bar{U} \longrightarrow K\left(E_{1}\right)$ be an upper semicontinuous compact multi-valued mapping.

Definition 3.1. The mapping $F$ defined above is called an "admissible" multi-valued mapping if there exist a topological space $X$ and two continuous single-valued mappings $X \stackrel{p}{\longrightarrow} \bar{U}$ and $X \stackrel{q}{\longrightarrow} E_{1}$ which satisfy the following conditions:

1. $p$ surjective;

2. $q \circ p^{-1}(x) \subset F(x)$ for every $x \in \bar{U}$;

3. $p^{-1}(x)$ is acyclic for every $x \in \bar{U}$. 
We shall consider the following multi-valued mapping:

$$
\Phi=f-F: \bar{U} \longrightarrow K\left(E_{1}\right) .
$$

The multi-valued mapping $\Phi$ is called a "multi-valued vector field generated" by $F$. We suppose that $f(x) \notin F(x)$ for every $x \in \partial U$.

Consider the set $K \subset U$ defined by

$$
K=\{x \in U \mid \theta \in(f-F)(x)\} .
$$

We shall build and study a degree for this class of multi-valued vector fields. we start with the following lemma.

Lemma 3.2. Let $S$ be a closed subset in $\bar{U}$ such that $S \cap K=\emptyset$. Then there exists $\varepsilon>0$ such that, $\|f(x)-y\| \geq \varepsilon_{o}$ for every $x \in S$ and $y \in F(x)$.

This is a consequence of the fact that $f$ is proper and $F$ compact.

Suppose now, that $K \cap \partial U=\emptyset$. Then by lemma 3.2 there exists $\varepsilon_{o}>0$ such that $f(x) \notin U_{\varepsilon_{0}}(F(x))$ for every $x \in \partial U$. Let $D=F(\bar{U})$ be a compact subset of $E_{1}$, choose in $D$ a finite $\frac{\varepsilon_{0}}{2}$ net with vertices $y_{1}, y_{2} \ldots y_{k}$, and consider the projector of Schauder $p: D \longrightarrow \overline{c o n} \bar{v}\left\{y_{1}, y_{2} \ldots y_{k}\right\}$ such that $\|y-p(y)\|<\frac{\varepsilon_{0}}{2}$ for every $y \in D$ Let $E_{p}=L\left(y_{1}, y_{2} \ldots y_{k}\right)$ be the linear vector space hull of the $\frac{\varepsilon_{0}}{2}$ net in D.

We can consider now the multi-valued mapping $F_{p}=p \cdot F: \bar{U} \longrightarrow K\left(E_{p}\right)$. In this case we have $F_{p}(x) \subset U_{\frac{\varepsilon_{0}}{2}} F(x)$, so $\theta \notin f(x)-F(x)$ for all $x \in \partial U$. Moreover, $K_{p} \cap \partial U=\emptyset$ where $K_{p}^{2}=\left\{x \in U \mid \theta \in\left(f-F_{p}\right)(x)\right\}$.

Using the theorem of Sapronov [16] concerning the decomposition of space we see that there exists a direct decomposition of $E_{1}=\tilde{Y}_{p} \oplus Y_{p}$ (where $\tilde{Y}_{p}$ is finite dimensional subspace) and a neighborhood $U\left(K_{p}\right)$ of $K_{p}$ such that $\pi \circ f: U\left(K_{p}\right) \longrightarrow Y_{p}$ is a submersion in the elements of $K_{p}$. The mapping $\pi$ is the natural projector which activates in parallel to $\tilde{Y}_{p}$.

Let us consider also the finite dimensional subspace $\widetilde{R}_{p}=E_{p}+\tilde{Y}_{p}, \pi_{p}$ : $E_{1} \longrightarrow R_{p}$, the natural projector which activates in parallel to $\widetilde{R}_{p}$ on the complementary subspace $R_{p}$ of the space $E_{1}$. Then $\pi_{p} \circ f: U\left(K_{p}\right) \longrightarrow R_{p}$ is also a submersion. Let be $M^{n}=f^{-1}\left(\widetilde{R}_{p}\right) \cap U\left(K_{p}\right)$. This is a n-dimensional oriented manifold of class $C^{1}$ and $n=\operatorname{dim} \widetilde{R}_{p}$, which is a consequence of the fact that $f \in \Phi_{0} C^{1}$ and

$$
M^{n}=\left(\pi_{p} \circ f\right)^{-1}(\theta)=f^{-1}\left(\tilde{R}_{p}\right) \cap U\left(K_{p}\right) .
$$

Similarly, we can built an oriented manifold $M^{n}$ of class $C^{1}$ and a finitedimensional approximation $\Phi_{p}=f-F_{p}$ of the multi-valued vector field $\Phi$ such that $\Phi_{p}: M^{n} \longrightarrow K\left(\widetilde{R}_{p}\right)$ and $K_{p} \subset M^{n}$.

Lemma 3.3. The multi-valued mapping $\Phi_{p}$ is an admissible multi-valued vector field.

The proof is a natural consequence of the construction of $\Phi_{p}$. 
Definition 3.4. The set of integers

$$
\operatorname{Deg}_{\theta}(f-F, \bar{U})=\left\{\left|\gamma_{\theta}\left[(\tilde{l}, \tilde{\varphi}), M^{n}\right]\right| \quad \mid \varphi=f \circ l-p \circ q,(l, q) \subset F\right\},
$$

is called the "local degree" of the multi-valued field $\Phi=f-F$. Here, $Z_{p}=$ $l^{-1}\left(M^{n}\right)$ and $\tilde{l}=\left.l\right|_{z_{p}}, \tilde{\varphi}=\left.\varphi\right|_{Z_{p}}$.

We should note that, in the case when $F$ is a multi-valued mapping with acyclic images, $\operatorname{Deg}_{\theta}(f-F, \bar{U})$ is a singleton:

$$
\operatorname{Deg}_{\theta}(f-F, \bar{U})=\left\{\left|\gamma_{\theta}(\tilde{t}, f \circ \tilde{t}-p \circ \tilde{r})\right|\right\}
$$

where $\tilde{t}$ is the projector of the graph $\Gamma_{M^{n}}(F)$ on $M^{n}, \tilde{r}$ is the projector of $\Gamma_{M^{n}}(F)$ on $E_{1}$ and $p$ is the projector of Schauder. We shall prove that the local degree $\operatorname{Deg}_{\theta}(f-F, \bar{U})$ of Definition 3.4 is well-defined.

Lemma 3.5. The local degree $\operatorname{Deg}_{\theta}(f-F, \bar{U})$ is independent of the choice of the subspace $\tilde{Y}_{p}$.

Proof. Let $\tilde{Y}_{p_{1}}$ and $\tilde{Y}_{p_{2}}$ be two finite-dimensional subspaces in $E_{1}$ with $E_{1}=\tilde{Y}_{p_{i}} \oplus Y_{p_{i}}, \quad i=1,2$, and let $\pi_{i} \circ f: U\left(K_{p}\right) \longrightarrow Y_{p_{i}}$ be the submersions. Suppose first that $\tilde{Y}_{p_{1}} \subset \tilde{Y}_{p_{2}}$ and consider the sequence of subsets connecting them:

$$
\tilde{Y}_{p_{1}}=\tilde{Y}_{0} \subset \tilde{Y}_{1} \subset \ldots . \subset \tilde{Y}_{s}=\tilde{Y}_{p_{2}}
$$

with $\operatorname{dim} \tilde{Y}_{j}-\operatorname{dim} \tilde{Y}_{j-1}=1$. Consider $R_{j}=E_{p}+\tilde{Y}_{j}$ and $T_{j}=f^{-1}\left(R_{j}\right) \cap$ $U\left(K_{p}\right)$. Evidently $T_{j-1}$ is a submanifold of class $C^{1}$ oriented in $T_{j}$, and $K_{p} \subset T_{0}=M^{p_{1}}$. Let $(l, q) \subset F$ be a selected $*$-pair of $F: \bar{U} \stackrel{l}{\longleftarrow} M \stackrel{q}{\longrightarrow} E_{1}$. Consider the sequence of $*$-pairs $\left(l_{j} ; \varphi_{j}\right)$ :

$$
T_{j} \stackrel{l_{j}}{\longleftarrow} M_{j} \stackrel{\varphi_{j}}{\longrightarrow} R_{j}, \quad j=0,1, \ldots, s,
$$

where $M_{j}=l^{-1}\left(T_{j}\right)$ and $l_{j}, \varphi_{j}$ are respectively the restriction of $l$ and $\varphi=$ $f_{\circ} l-p_{\circ} q$ on $M_{j}$. In this case $\left(l_{j}, \varphi_{j}\right) \subset \Phi_{j}$, where $\Phi_{j}$ is the restriction of $f-p \circ F$ on $M_{j}$. Now, using the property of the restriction we have

$$
\left|\gamma_{\theta}\left[\left(l_{0}, \varphi_{0}\right), T_{0}\right]\right|=\left|\gamma_{\theta}\left[\left(l_{1}, \varphi_{1}\right), T_{1}\right]\right|=\ldots=\left|\gamma_{\theta}\left[\left(l_{s}, \varphi_{s}\right), T_{s}\right]\right| .
$$

In the case when $\tilde{Y}_{p_{1}} \not \subset \tilde{Y}_{p_{2}}$, we can consider $\tilde{Y}_{p_{1}}+\tilde{Y}_{p_{2}}=\tilde{Y}_{p}$. This space satisfies the condition of the theorem of Sapronov [16], and $\tilde{Y}_{p_{1}} \subset \tilde{Y}_{p}, \tilde{Y}_{p_{2}} \subset \tilde{Y}_{p}$ , so we can use the above part of this proof.

Lemma 3.6. The local degree $\operatorname{Deg}_{\theta}(f-F, \bar{U})$ is independent of the choice of the projector of Schauder.

Proof. Let $E_{p_{1}}$ and $E_{p_{2}}$ be two finite-dimensional subspaces. Consider $p_{1}$ : $D \longrightarrow E_{p_{1}}$ and $p_{2}: D \longrightarrow E_{p_{2}}$ the Schauder projectors with $\left\|x-p_{i}(x)\right\|<\frac{\varepsilon_{0}}{2}$ for every $x \in D$.

We can define two multi-valued mappings $F_{p_{1}}=p_{1} \circ F$ and $F_{p_{2}}=p_{2} \circ F$. 
Let $E_{p_{0}}=E_{p_{1}}+E_{p_{2}}$. Then $F_{p_{1}}: \bar{U} \longrightarrow E_{p_{0}}$ and $F_{p_{2} .}: \bar{U} \longrightarrow E_{p_{0}}$. Let $\tilde{Y}_{p}$ be a finite-dimensional subspace such that $E_{1}=\tilde{Y}_{p} \oplus Y_{p}$ and

$$
\pi \circ f: U\left(K_{p_{1}} \cup K_{p_{2}}\right) \longrightarrow Y_{p}
$$

the associated submersion. Then we can consider $R_{p_{1}}=E_{p_{1}}+\tilde{Y}_{p}, R_{p_{2}}=$ $E_{p_{2}}+\tilde{Y}_{p}$ and $R_{p_{0}}=E_{p_{0}}+\tilde{Y}_{p}$. Obviously, $R_{p_{1}}$ and $R_{p_{2}}$ are contained in $R_{p_{0}}$.

The spaces $M^{n_{i}}=f^{-1}\left(R_{p_{i}}\right) \cap U\left(K_{1} \cup K_{2}\right), i=0,1,2$, are manifolds such that $M^{n_{1}}$ and $M^{n_{2}}$ are two oriented submanifolds of $M^{n_{0}}$.

Let $\Phi_{p_{1}}=f-p_{1} \circ F$ and $\Phi_{p_{2}}=f-p_{2} \circ F$ be admissible multi-valued vector fields on the manifold $M^{n_{0}}$. Let $(l, q) \subset F$ be an admissible *-pair:

$$
\bar{U} \stackrel{l}{\longleftarrow} Z \stackrel{q}{\longrightarrow} E_{1} \text {. }
$$

Let $Z_{0}=l^{-1}\left(M^{n_{0}}\right)$. Then we can consider the $*$-pairs $\left(l ; \varphi_{1}\right),\left(l ; \varphi_{2}\right)$ such that: $\varphi_{i}=f \circ l-p_{i} \circ q, i=1,2$, defined by

$$
\left(M^{n_{0}}, M^{n_{0}} \backslash\left(K_{p_{1}} \cup K_{p_{2}}\right)\right) \stackrel{l}{\longleftarrow}\left(Z_{0}, Z_{0} \backslash l^{-1}\left(K_{p_{1}} \cup K_{p_{2}}\right)\right) \stackrel{\varphi_{i}}{\longrightarrow}\left(R_{p_{0}}, R_{p_{0}} \backslash \theta\right)
$$

for $i=1,2$. Since

$$
\left\|x-\lambda p_{1}(x)-(1-\lambda) p_{2}(x)\right\|<\frac{\varepsilon_{0}}{2}, \quad x \in D, \lambda \in[0,1],
$$

we see that the mappings

$$
\varphi_{1}, \varphi_{2}:\left(Z_{0}, Z_{0} \backslash l^{-1}\left(K_{p_{1}} \cup K_{p_{2}}\right)\right) \longrightarrow\left(R_{p_{0}}, R_{p_{0}} \backslash \theta\right)
$$

are homotopic. So, $\gamma_{\theta}\left[\left(l, \varphi_{1}\right), M^{n_{0}}\right]=\gamma_{\theta}\left[\left(l, \varphi_{2}\right), M^{n_{0}}\right]$.

We must prove now that

$$
\left|\gamma_{\theta}\left[\left(l, \varphi_{1}\right), M^{n_{0}}\right]\right|=\left|\gamma_{\theta}\left[\left(l, \varphi_{1}\right), M^{n_{1}}\right]\right|
$$

and

$$
\left|\gamma_{\theta}\left[\left(l, \varphi_{2}\right), M^{n_{0}}\right]\right|=\left|\gamma_{\theta}\left[\left(l, \varphi_{2}\right), M^{n_{2}}\right]\right| .
$$

Let us consider the sequence of subspaces connecting the subspaces $R_{p_{1}}$ and $R_{p_{0}}$ :

$$
R_{p_{1}}=L_{0} \subset L_{1} \subset \ldots \subset L_{s}=R_{p_{0}},
$$

with $\operatorname{dim} L_{j}-\operatorname{dim} L_{j-1}=1, j=1,2, \ldots, s$.

So we have the sequence of oriented submanifolds of class $C^{1}$ :

$$
T_{0} \subset T_{1} \subset \ldots \subset T_{s-1} \subset T_{s},
$$

where $T_{j}=f^{-1}\left(L_{j}\right) \cap U\left(K_{1} \cup K_{2}\right) ; T_{0}=M^{n_{1}}, T_{s}=M^{n_{0}}$.

Let $Z_{j}=l^{-1}\left(T_{j}\right), j=1,2, \ldots, s$. Then we have the admissible $*$-pairs $\left(l_{j}, \psi_{j}\right), j=1,2, \ldots, s$, defined by:

$$
T_{j} \stackrel{l_{j}}{\longleftarrow} Z_{j} \stackrel{\Psi_{j}}{\longrightarrow} L_{j}
$$

where $l_{j}$ is the restriction of $l$ on $Z_{j}$ and $\psi_{j}$ the restriction of $\varphi_{1}$ on $L_{j}$.

On the other hand $l_{i} \circ \psi_{i}^{-1}(\theta)=l_{j} \circ \psi_{j}^{-1}(\theta)=K_{p_{1}}$ for every $i, j$. Then using the proposition of the restriction we obtain

$$
\left|\gamma_{\theta}\left(l_{0}, \psi_{0}\right)\right|=\left|\gamma_{\theta}\left(l_{1}, \psi_{1}\right)\right|=\cdots\left|\gamma_{\theta}\left(l_{s}, \psi_{s}\right)\right| \text {. }
$$


The absolute value is necessary from the orientations in $T_{j}$ which can be incompatible.

We shall now give some properties of this degree.

Proposition 3.7. If $\operatorname{Deg}_{\theta}(f-F, \bar{U}) \neq\{0\}$, then there exists $x_{0} \in U$ such that $\theta \in f\left(x_{0}\right)-F\left(x_{0}\right)$.

Proof. Suppose that $\theta \notin f(x)-F(x)$ for every $x \in \bar{U}$. Then using Lemma 3.2 we see that exists $\varepsilon_{o}>0$ with $\|x-y\| \geq \varepsilon_{o}$ if $x \in \bar{U}$ and $y \in F(x)$.

If $p$ is the projector of Schauder such that $\|x-p(x)\|<\frac{\varepsilon_{0}}{2}$ for $x \in D$, then $\Phi_{p}=f-p \circ F$ has no particular point. Thus, for every admissible $*$-pair $(l, q) \subset F$ we have $\gamma_{\theta}(l, f \circ l-p \circ q)=0$. So, $\operatorname{Deg}_{\theta}(f-F, \bar{U})=\{0\}$.

Let $\varepsilon_{o}=\min _{z \in \underset{x \in \partial U}{F}(x)}\|f(x)-z\|$. From the fact that $f$ is proper and $F$ is compact we can affirm that $\varepsilon_{o}>0$.

Proposition 3.8. For every $y$ with $\|y\|<\frac{\varepsilon_{o}}{2}$ we have

$$
\operatorname{Deg}_{\theta}(f-F, \bar{U})=\operatorname{Deg}_{y}(f-F, \bar{U}) \text {. }
$$

Proof. Let $p$ be the projector of Schauder associated with $\frac{\varepsilon_{0}}{2}$. We can suppose that the subspace $E_{p}$ contains the point $y$. Let $(l, q) \subset F$. Then $\varphi=f \circ l-$ $p \circ q: Z \longrightarrow E_{1}$. From the definition of the local degree we have the $*$-pair

$$
M^{n} \stackrel{\tilde{l}}{\longleftarrow} Z_{p} \stackrel{\tilde{\varphi}}{\longrightarrow} \widetilde{R}_{p}=E_{p}+\tilde{Y}_{p}
$$

From the fact that the mapping $f$ is proper we can deduce that $\tilde{l} \circ(\tilde{\varphi})^{-1}(y)$ is compact. So $\gamma_{\theta}(\tilde{l}, \tilde{\varphi})=\gamma_{y}(\tilde{l}, \tilde{\varphi})$. This fact is a consequence of Proposition 1.12. So, $\operatorname{Deg}_{\theta}(f-F, \bar{U})=\operatorname{Deg}_{y}(f-F, \bar{U})$.

Definition 3.9. Let $\Phi(t, x)=f(x)-F(t, x): \bar{U} \times[0,1] \longrightarrow K\left(E_{1}\right)$. We say that $\Phi$ is a "homotopy" if the following two conditions hold.

1. F is a compact, upper semicontinuous and admissible multi-valued mapping;

2. $\theta \notin \Phi(t, x)$ for every $t \in[0,1]$ and $x \in \partial U$.

Proposition 3.10. Let $\Phi$ be a homotopy. Then

$$
\operatorname{Deg}_{\theta}(\Phi(0, \cdot), \bar{U}) \cap \operatorname{Deg}_{\theta}(\Phi(1, \cdot), \bar{U}) \neq \emptyset \text {. }
$$

Proof. Let $D=\left\{y \in E_{1} \mid \exists(t, x) \in[0,1] \times \bar{U} ; y \in F(t, x)\right\}$ and let $\varepsilon_{o}>0$ be such that $\|x-y\| \geq \varepsilon_{o}$ for $y \in F(t, x),(t, x) \in[0,1] \times \partial U$. Let $p: D \longrightarrow E_{1}$ be the projector of Schauder such that $\|x-p(x)\|<\frac{\varepsilon_{0}}{2}$ for $x \in D$.

As in the definition of the local degree, consider the finite-dimensional space $\widetilde{R}_{p} \supset p(D)$ and $M^{n}=f^{-1}\left(\widetilde{R}_{p}\right) \cap U\left(K_{p}\right)$, where $K_{p}=\{x \in U \mid \theta \in$ $f(x)-p \circ F(t, x), t \in[0,1]\}$. We can define on $M^{n}$ the homotopy $\Phi_{p}(t, x)=$ $f(x)-p \circ F(t, x)$. Now the proof is a consequence of the proposition of homotopy invariance. 
The nonlinear Fredholm mappings $f$ are also admissible multi-valued vector fields. For this class we can define a local degree $\operatorname{Deg}_{\theta}(f, \bar{U})$. On the other hand, a degree, $\gamma_{\theta}(f, \bar{U})$, for nonlinear Fredholm mappings has been defined in [6]. We can prove that

$$
\operatorname{Deg}_{\theta}(f, \bar{U})=\left\{\left|\gamma_{\theta}(f, \bar{U})\right|\right\} .
$$

As in the case of the usual degree, the local degree $D e g_{\theta}(\Phi, \bar{U})$ can be used to solve the existence problem for the inclusion $\theta \in \Phi(x)$. For example let us give the following proposition. Let $U$ be an open bounded subset of $E, f: \bar{U} \longrightarrow$ $E_{1}, F: \bar{U} \longrightarrow K\left(E_{1}\right)$.

Proposition 3.11. Let $f \in \Phi_{0} C^{1}$ be proper, let $F$ be a compact upper semicontinuous admissible multi-valued mapping. Suppose that

1. $\|f(x)\| \geq\|y\|$ for every $y \in F(x), x \in \partial U$;

2. $\|f(x)\| \neq 0$ for every $x \in \partial U$;

3. $\operatorname{Deg}_{\theta}(f, \bar{U}) \neq 0$.

Then there exists an element $x_{0} \in U$ such that $\theta \in f\left(x_{0}\right)-F\left(x_{0}\right)$.

Proof. In the case when $\theta \in f(x)-F(x)$ for some $x \in \partial U$ the proposition is proved. Suppose that $\theta \notin f(x)-F(x)$ for every $x \in \partial U$. Then we can consider the homotopy $\Phi(\lambda, x)=f(x)-\lambda F(x)$. It is easy to see that $\theta \notin$ $\Phi(\lambda, x)$ on $(\lambda, x) \in[0,1] \times \partial U$. From 3.10, $\operatorname{Deg}_{\theta}(\Phi(0, \cdot), \bar{U}) \cap \operatorname{Deg}_{\theta}(\Phi(1, \cdot), \bar{U}$ $\neq \emptyset$. Since $\Phi(0, \cdot)=f(\cdot)$ and $\operatorname{Deg}_{\theta}(f, \bar{U}) \neq 0$, we can deduce that $\operatorname{Deg}_{\theta}(f-$ $F, \bar{U}) \neq\{0\}$. Then there exists an element $x_{0} \in U$ such that $\theta \in f\left(x_{0}\right)-$ $F\left(x_{0}\right)$.

\section{Some Applichtions of the LOCAL DEGREE}

In this section we shall consider the existence solutions of a class of operator inclusions. For this purpose we consider the following hypotheses.

Let $W$ be a bounded open subset of $R^{n}$ and let $f:[0, h] \times \bar{W} \longrightarrow R^{n}$ and $g:[0, h] \times R^{n} \longrightarrow R^{n}$ be two single-valued continuous mappings. Let $F:[0, h] \times \bar{W} \longrightarrow K_{V}\left(R^{n}\right)$ be a multi-valued upper semicontinuous mapping which satisfies the Caratheodory conditions:

1. For every $x \in E$ the multi-valued mapping $F(\cdot, x):[0, h] \longrightarrow K_{V}\left(R^{n}\right)$ is measurable.

2. For almost all $t \in[0, h]$ the multi-valued $F(t, \cdot): \bar{W} \longrightarrow K_{V}\left(R^{n}\right)$ is upper semicontinuous .

3. There exist two summable single-valued mappings $\alpha, \beta:[0, h] \longrightarrow R$ such that

$$
\|F(t, x)\|=\sup _{y \in F(t, x)}\|y\| \leq \alpha(t)+\beta(t)\|x\| .
$$

Let us consider the following system:

$$
\begin{aligned}
& y^{\prime}(t) \in F(t, x(t)), \\
& y(0)=0, \\
& f(t, x(t))=\varepsilon g(t, y(t)),
\end{aligned}
$$


where $\varepsilon>0$ is a given number.

The solution of the system (1) on the interval $[0, h]$ is defined by a pair of continuous single mappings $(x(\cdot), y(\cdot))$, where $x, y:[0, h] \longrightarrow R^{n}$ are such that

1. $y(\cdot)$ is an absolutely continuous mapping, $y^{\prime}(t) \in F(t, x(t))$ for almost all $t \in[0, h]$ and $y(0)=0$;

2. $f(t, x(t))=\varepsilon g(t, y(t))$ for every $t \in[0, h]$.

We shall study the existence of the solution of the system (1).

It is simple to see that the problem (1), with the right choice of mappings $f$ and $g$, is a generalization of the Cauchy problem and a large class of boundary value problems for the differential inclusions.

Let $U=\left\{u \in C_{[0, h]} \mid u(t) \in W\right.$, for every $\left.t \in[0, h]\right\}$. This set is a bounded open subset of $C_{[0, h]}$. We can define the nonlinear operator f $: \bar{U} \longrightarrow C_{[0, h]}$ which is called the operator of superposition. This operator is defined by $\mathbf{f}(u)(t)=f(t, u(t))$ for every $u \in \bar{U}$ and $t \in[0, h]$. In the same way, from the mapping $g$ we can define $\mathbf{g}: C_{[0, h]} \longrightarrow C_{[0, h]}$.

Let us now consider the multi-valued mapping :

$K(u)(t)=\left\{\int_{0}^{t} y(\tau) d \tau \mid y(\tau) \in F(\tau, u(\tau))\right.$ almost everywhere $\left.\tau \in[0, h]\right\}$,

where $u \in \bar{U}$ and $t \in[0, h]$.

Proposition 4.1. The operator $K$ is upper semicontinuous and has nonempty compact convex images, i.e.,

$$
K: \bar{U} \longrightarrow K_{V}\left(C_{[0, h]}\right) .
$$

For the proof of the proposition see [8], [1].

We can consider now the following operator inclusion:

$$
\theta \in \mathbf{f}(u)-\varepsilon(\mathbf{g} \circ K)(u)
$$

The solution of the inclusion (2) is a continuous mapping $u_{0}:[0, h] \longrightarrow R^{n}$ such that

for every $t \in[0, h]$.

$$
\theta \in \mathbf{f}\left(u_{0}\right)(t)-\varepsilon(\mathbf{g} \circ K)\left(u_{0}\right)(t)
$$

Proposition 4.2. The problems (1) and (2) are equivalent.

Proof. Let $\left(x_{0}(\cdot), y_{0}(\cdot)\right)$ be a solution of System (1). Then $f\left(t, x_{0}(t)\right)=$ $\varepsilon g\left(t, \int_{0}^{t} y_{0}^{\prime}(\tau) d \tau\right)$, where $y_{0}^{\prime}(\tau) \in F\left(\tau, x_{0}(\tau)\right)$ for almost everywhere $\tau \in$ $[0, h]$. So, we can deduce that $\mathbf{f}\left(x_{0}\right)(t) \in \varepsilon(\mathbf{g} \circ K)\left(x_{0}\right)(t)$, and this means that $x_{0}$ is a solution of the problem (2).

Consider now $u_{0}$ a solution of the problem $(2)$. Then $\mathbf{f}\left(u_{0}\right)(t) \in$ $\varepsilon(\mathbf{g} \circ K)\left(u_{0}\right)(t)$. This signifies that there exists a mapping $z$ such that $z(\tau) \in$ $F\left(\tau, u_{0}(\tau)\right)$ for almost everywhere $\tau \in[0, h]$ and $f\left(t, u_{0}(t)\right) \in \varepsilon g\left(t, \int_{0}^{t} z(\tau)\right.$ 
$d \tau)$. Then if $y_{0}(t)=\int_{0}^{t} z(\tau) d \tau$ the pair $\left(x_{0}(\cdot), y_{0}(\cdot)\right)$ is a solution of the problem (1).

Let us now consider the solvability of the problem (2). For this purpose we shall make the following hypotheses.

1. The mapping $f:[0, h] \times W \longrightarrow R^{n}$ is continuous. Moreover, for $(t, v) \in$ $[0, h] \times W, f$ has continuous partial derivatives with respect to the vector variable $v$.

2. The Jacobian matrix $f_{v}^{\prime}(t, v)$ satisfies $\operatorname{det}\left[f_{v}^{\prime}(t, v)\right] \neq 0$ for every $t \in$ $[0, h]$ and $v \in W$.

From this condition we can deduce that the single-valued mapping $f_{t}=f(t, \cdot): W \longrightarrow R^{n}$ is a local homeomorphism for every $t \in[0, h]$.

3. The single-valued mapping $f_{t}=f(t, \cdot): W \longrightarrow R^{n}$ is a homeomorphism for every $t \in[0, h]$ on it range of values.

In [15] we can find some properties for functions $f_{t}$ which satisfy the above assumption.

Proposition 4.3. The operator of superposition $\mathbf{f}: U \longrightarrow C_{[0, h]}$ is Fréchetdifferentiable and is a nonlinear Fredholm operator of index zero.

The proof of this proposition is a consequence of the fact that the Fréchetderivative $\mathbf{f}^{\prime}(u)$ of the operator of superposition $\mathbf{f}$ is an isomorphism for every $u \in U$.

Proposition 4.4. The operator of superposition $\mathbf{f}$ is a homeomorphism on it's range of values.

Proof. To show that $\mathbf{f}$ is an injective mapping, suppose that there exits a function $y \in \mathbf{f}(U)$ such that $\mathbf{f}(x)=y, \mathbf{f}\left(x_{1}\right)=y$ and, for some $t_{0} \in$ $[0, h]$, we have $x\left(t_{0}\right) \neq x_{1}\left(t_{0}\right)$. Then, using Condition 3 , we can deduce that $f\left(t_{0}, x\left(t_{0}\right)\right) \neq f\left(t_{0}, x_{1}\left(t_{0}\right)\right)$. But $y\left(t_{0}\right)=f\left(t_{0}, x\left(t_{0}\right)\right)=f\left(t_{0}, x_{1}\left(t_{0}\right)\right)$, from which we obtain the desired contradiction.

The mapping $\mathbf{f}^{-1}$ is continued because $\mathbf{f}$ is a local homeomorphism.

Proposition 4.5. The operator of superposition $\mathbf{f}$ is proper.

The proof is a consequence of the proposition 4.4 .

Proposition 4.6. The mapping $\mathbf{G}=\mathbf{g} \circ K: \bar{U} \longrightarrow K\left(C_{[0, h]}\right)$ is an admissible, compact, upper semicontinuous and multi-valued mapping.

Proof. Let $\Gamma_{K}$ be the graph of the multi-valued mapping $K$, and let $(t, r)$ be the pair composed of the natural projectors $t: \Gamma_{K} \longrightarrow \bar{U}$ and $r: \Gamma_{K} \longrightarrow$ $C_{[0, h]}$. Then the pair of single-valued mappings $(t, g \circ r)$ is a selected $*$ - pair of the multi-valued mapping $\mathbf{G}$. On the other hand, $t^{-1}(x)$ is acyclic because this set is convex.

Theorem 4.7. Let the above conditions on the mappings $f, g, F$ be satisfied. Assume further that the following conditions hold. 
1. $\theta \in f(t, W)$ for every $t \in[0, h]$;

2. $\theta \notin f(t, y)$ for every $t \in[0, h], y \in \partial W$.

Then there exists $\varepsilon_{o}>0$ such that for every $\varepsilon \in\left[0, \varepsilon_{o}\right]$ the system (1) has a solution.

Proof. Let us prove that the operator inclusion (2) has a solution in $U$. From the fact that the single-valued mapping $f$ is continuous on the compact subset $[0, h] \times \partial W$ and is not null in $[0, h] \times \partial W$, there exists $\delta>0$ such that $U_{\delta}(\theta) \cap f([0, h] \times \partial W)=\emptyset$. Then for every $u \in \partial U$ we have $\|\mathbf{f}(u)\| \geq \delta$. Since the multi-valued mapping $\mathbf{G}$ is upper semicontinuous and compact there exists $N>0$ such that $\|z\| \leq N$ for every $z \in \mathbf{G}(\bar{U})$.

Consider $0<\varepsilon_{o}<\frac{\delta}{N}$ Then $\theta \notin \mathbf{f}(x)-\varepsilon \mathbf{G}(x)$ for every $x \in \partial U$ and $\varepsilon \in\left[0, \varepsilon_{o}\right]$. Consider now the homotopy $\Phi(\lambda, x)=\mathbf{f}(x)-\varepsilon \lambda \mathbf{G}(x)$. It is easy to see that $\theta \notin \Phi(\lambda, x)$ for every $\lambda \in[0,1]$ and $x \in \partial U$, and, consequently, $\operatorname{Deg}_{\theta}(\Phi(0, \cdot), \bar{U}) \cap \operatorname{Deg}_{\theta}(\Phi(1, \cdot), \bar{U}) \neq \emptyset$. Considering that $\Phi(0, \cdot)=\mathbf{f}(\cdot)$ and the fact that $\mathbf{f}$ is a homeomorphism and $\theta \in \mathbf{f}(U)$, we can deduce that $\operatorname{Deg}_{\theta}(\mathbf{f}, \bar{U})=\{1\}$ (see $\left.[6]\right)$. So, $1 \in \operatorname{Deg}_{\theta}(\mathbf{f}-\varepsilon \mathbf{G}, \bar{U})$. Then from the proposition we can deduce that there exists a solution for the operator inclusion (2).

Now we consider the problems (1) and (2) when $\varepsilon=1$.

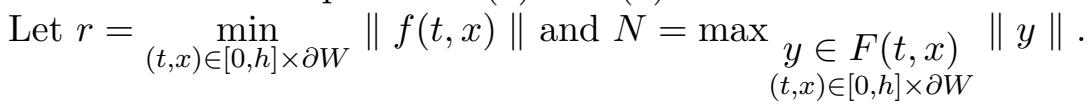

Theorem 4.8. If the conditions Theorem 4.7 are satisfied and $N<r$, then the operator inclusion

$$
\theta \in \mathbf{f}(u)-(\mathbf{g} \circ K)(u)
$$

has a solution.

The proof of this theorem is similar to the preceding one.

\section{REFERENCES}

[1] J-P. Aubin and A. Cellina, Differential Inclusions. Set valued maps and viability theory, Grundlehren der Mathematischen Vissenshaften, \#264, Springer-Verlag, BerlinNew York, 1984.

[2] N. Benkafadar, B. D. Gel'man, On a local degree for multi-valued mappings with a principal part a Fredholm mapping, VINITI, \#3422-82, 1982 (in Russian).

[3] Y. G. Borisovich, Topological degree for Fredholm maps perturbed by multi-valued operators, VINITI, \#5026-80, 1980 (in Russian).

[4] Y. G. Borisovich, Modern approach to the theory of topological characteristics of nonlinear operators, I, Lecture Notes in Math., \#1334, Springer-Verlag, Berlin-New York, 1988, 199-220.

[5] Y. G. Borisovich, Modern approach to the theory of topological characteristics of nonlinear operators, II,Lecture Notes in Math., \#1453, Springer-Verlag, Berlin-New York, 1990, 21-50.

[6] Y. G. Borisovich, B. D. Gel'man, A. D. Myshkis and V. V. Obukhovsky, Topological methods in the theory of fixed points of multi-valued mappings Uspekhi. Mat. Nauk, 35 (1980), 59-126 (in Russian).

[7] Y. G. Borisovich, B. D. Gel'man, A. D. Myshkis and V. V. Obukhovsky, Multi-valued mappings, VINITI, 19 (1982), 127-230 (in Russian). 
[8] Y. G. Borisovich, B. D. Gel'man, A. D. Myshkis and V. V. Obukhovsky, Introduction to the Theory of Multi-valued Mappings, Voronezh Gos. Univ., Voronezh, 1986 (in Russian).

[9] Y. G. Borisovich, V. G. Zvyagin and Y. I. Sapronov, Nonlinear Fredholm mappings and Leray-Schauder theory, Uspekhi. Mat. Nauk, 32 (1977), 3-54 (in Russian).

[10] A. Dold, Lectures on Algebraic Topology, Springer-Verlag, Berlin-New York, 1972.

[11] L. Gorniewicz, Homological methods in fixed point theory of multi-valued maps, Dissertationes Math. (Rozprawy Mat.), \#129, Warsaw, 1976.

[12] M. W. Hirsch, Differential Topology, Springer-Verlag, Berlin-New York, 1976.

[13] Z. Kucharski, A coincidence index, Bull. Acad. Polon. Sci. Sér. Sci. Math. Astronom. Phys. 24 (1976), 245-252.

[14] J. R. Munkres, Elementary Differential Topology, Princeton University Press, 1963.

[15] J. M. Ortega, W. C. Rheinboldt, Iterative Solution of Nonlinear Equations in Several Variables, Academic Press,1970.

[16] Y. I. Sapronov, A local inverse for a nonlinear Fredholm map, Funct. Anal. 5 (1971), 38-43 (in Russian).

[17] E. H. Spanier, Algebraic Topology, McGraw-Hill, 1966.

Institut de Mathématiques, Université de Constantine, Route de Ain ElBey, 25000 Constantine, ALGERIE

Mathematics Faculty, Voronezh State University, Universitetskaya Pl.1, 394693 VORONEZH, RUSSIA

E-mail address: root@mathd.vucnit.voronezh.su 


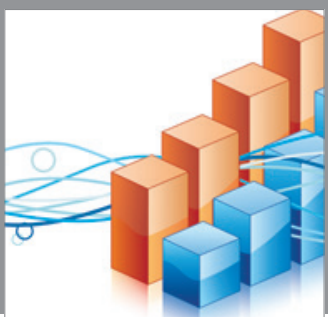

Advances in

Operations Research

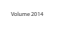

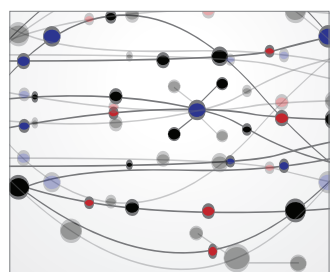

\section{The Scientific} World Journal
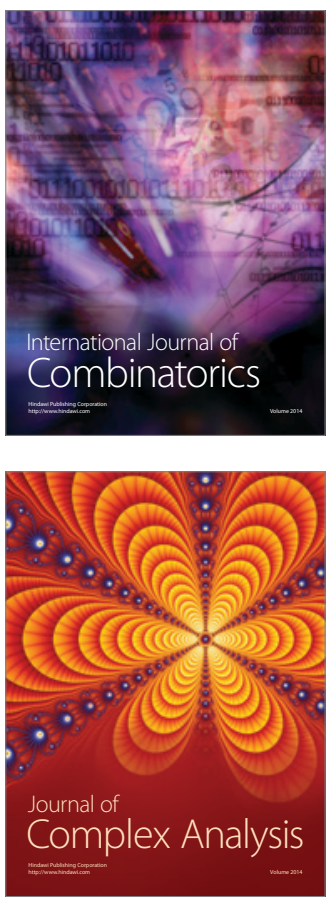

International Journal of

Mathematics and

Mathematical

Sciences
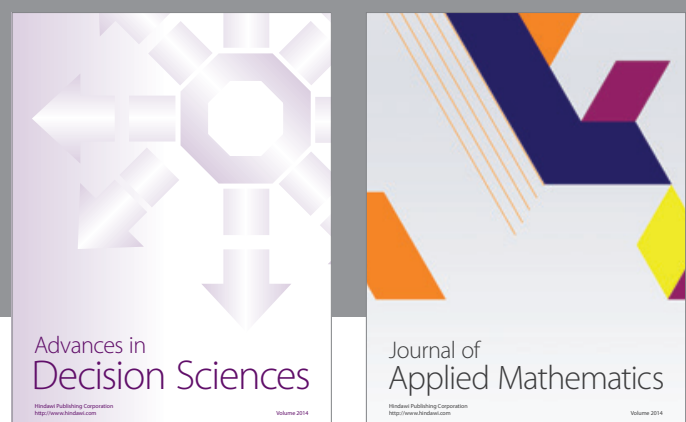

Journal of

Applied Mathematics
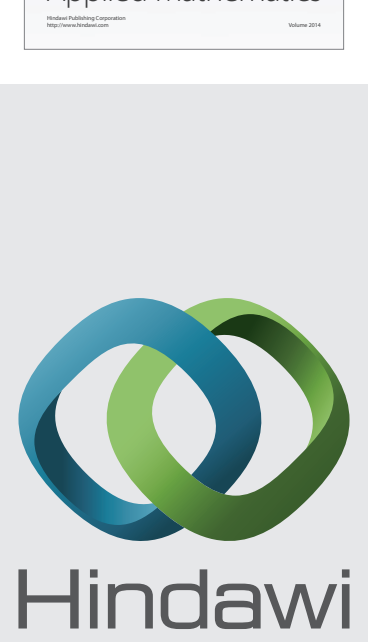

Submit your manuscripts at http://www.hindawi.com
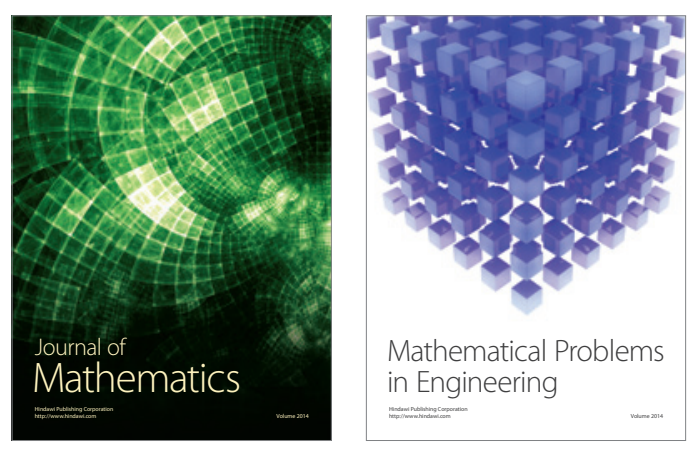

Mathematical Problems in Engineering
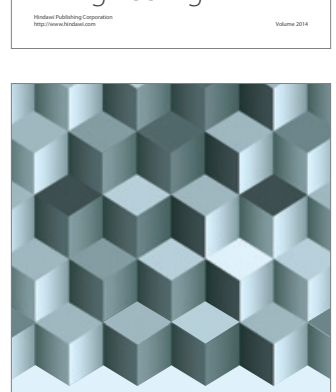

Journal of

Function Spaces
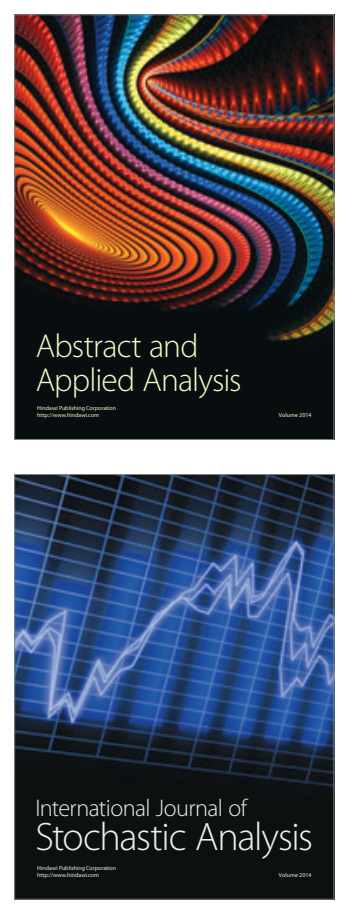

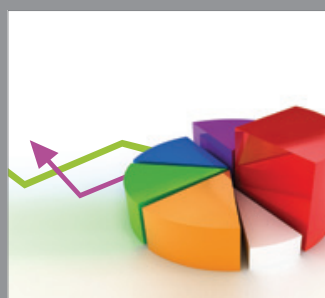

ournal of

Probability and Statistics

Promensencen
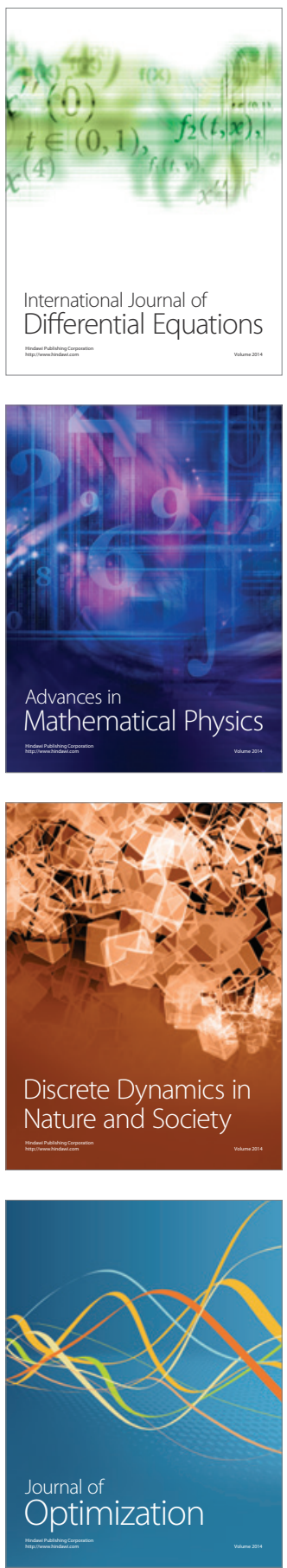